\title{
Resuscitation time bias and the apparent benefit of early interventions in out-of-hospital cardiac arrest: a review of the literature
}

Johannes von Vopelius-Feldt, PhD MSC MRCEM DipIMC

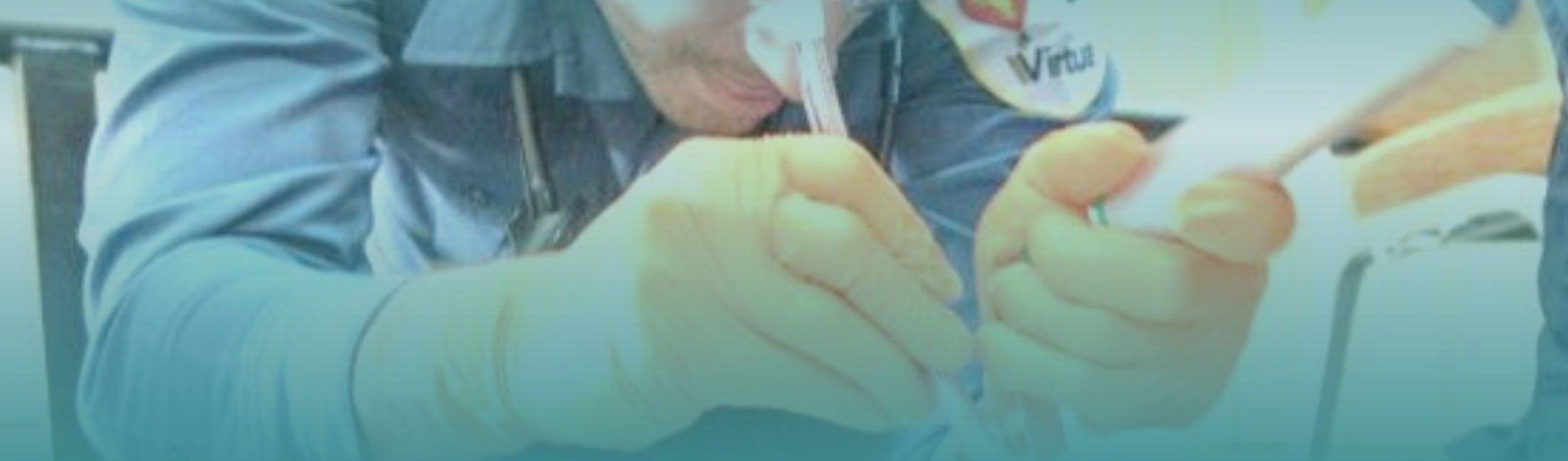

Background Despite a lack of clear evidence for advanced airway management (AAM) during cardiac arrest, a number of observational studies suggest that early AAM is associated with higher rates of return of spontaneous circulation (ROSC), survival to hospital discharge and favourable neurological outcomes. However, these findings might be confounded by resuscitation time bias (RTB).

Methods We undertook a focused review of the literature relevant to the timing of AAM during adult cardiac arrest, using relevant search terms in PubMed, limited to articles in English and during the last 10 years. Each included article was examined for how well its methods controlled for RTB.

\section{Illustration of resuscitation time bias: AAM within $20 \mathrm{~min}$ or later}

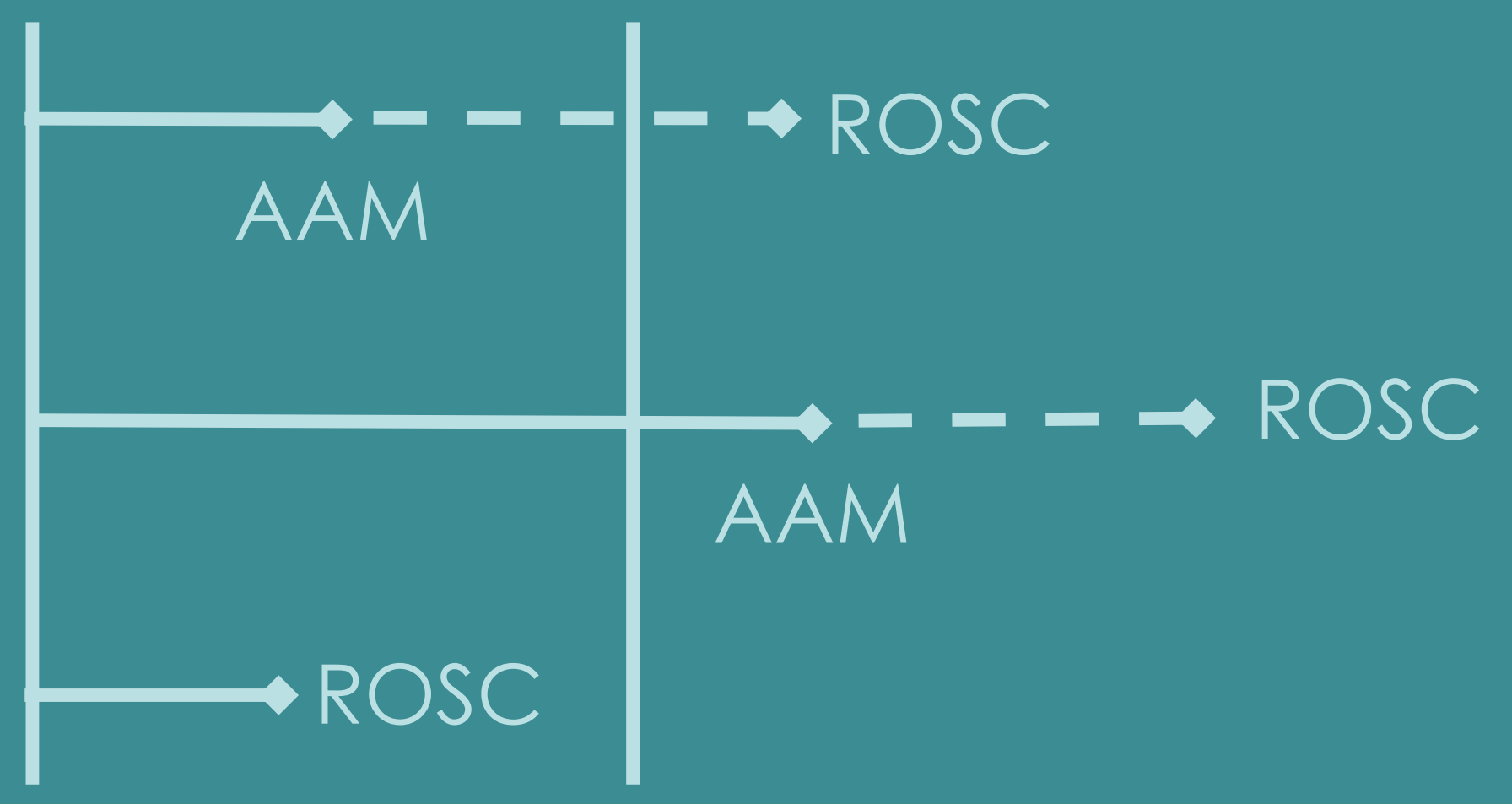

ROSC can occur anytime after early AAM higher probability of good outcome with early AAM

If AAM occurs late, earliest possibility of ROSC is affer $20 \mathrm{~min}$ - lower probability of good outcome

ROSC occurs prior to AAM - higher probability of good outcome but not Omin $20 \mathrm{~min}$ included in studies as no AAM performed

Results Of the eight studies included in this review, only one study used appropriate statistical methods to adjust for RTB. The other seven studies were all of observational design and demonstrated an association between early AAM and a range of favourable outcomes. All were deemed to be at risk of over-estimating the benefit of early AAM due to incomplete or lack of adjustment for RTB. The one study which used risk set matching to fully adjust for RTB demonstrated significantly worse outcomes if AAM was undertaken within the first $15 \mathrm{~min}$ of cardiac arrest.

Discussion The currently available literature regarding the timing of AAM during OHCA is likely to overestimate any potential benefit from early AAM. Further research with appropriate adjustment or randomisation is required to address this question. 